\title{
Optimizing the Aspect Ratio of Cephalexin in Reactive Crystallization by Controlling Supersaturation and Seeding Policy
}

\author{
Mingchen $\mathrm{Li}^{1,2} \cdot$ Zeren Shang ${ }^{1,2} \cdot$ Baohong Hou ${ }^{1,2}$
}

Received: 18 August 2018 / Revised: 25 October 2018 / Accepted: 29 November 2018 / Published online: 12 February 2019

(c) The Author(s) 2019

\begin{abstract}
Batch crystallization in acidic aqueous solution of cephalexin was conducted by reactive crystallization with or without seeding. Supersaturation was generated by mixing ammonia and acidic aqueous solution of cephalexin and controlled by solution feeding rate and seeding conditions. UV and Morphologi G3 were used to measure supersaturation and aspect ratio. Experimental results demonstrated that burst nucleation occurred and the products were needle-like at high supersaturation; meanwhile, the products were plate-like and had high aspect ratio at low supersaturation. Analysis of the measured supersaturation profiles and corresponding aspect ratio explained the mechanisms governing the aspect ratio. The optimized operating parameters were also proposed (seeding supersaturation is equal to 1.3 , seed mass ratio $8 \%$ and feeding rate $368 \mu \mathrm{L} / \mathrm{min}$ ).
\end{abstract}

Keywords Reactive crystallization · Supersaturation control $\cdot$ Aspect ratio $\cdot$ Crystal habit

\section{Introduction}

Batch crystallization is widely used to produce high-value chemicals $[1,2]$. In industrial operation, product crystals with narrow crystal size distribution are preferred for better downstream operation efficiency and total separation economics than those with wide distribution [3].

Crystal shape is an important property which is desirable to be controlled through various steps to vary physicochemical properties. For example, filterability, dissolution behavior and conveying performance of crystallized solids are key factors for design of downstream processes and are influenced by crystal shape [4]. Therefore, scholars must focus on crystal habit during crystallization to obtain products with desired characteristics. The crystal shape can be

Electronic supplementary material The online version of this article (https://doi.org/10.1007/s12209-019-00185-z) contains supplementary material, which is available to authorized users.

Baohong Hou

houbaohong@tju.edu.cn

1 School of Chemical Engineering and Technology, State Key Laboratory of Chemical Engineering, Tianjin University, Tianjin 300350, China

2 Collaborative Innovation Center of Chemical Science and Engineering of Tianjin, Tianjin University, Tianjin 300350, China optimized by controlling supersaturation and other operation parameters. Supersaturation $S$ is defined as the ratio of the monomer concentration in solution to the equilibrium monomer concentration. Yang et al. [5] described a research on shape control of potassium dihydrogen phosphate in batch cooling crystallization by controlling supersaturation as a variable. Bordawekar et al. [6] investigated the influence of supersaturation on the crystal habit of choline fenofibrate to obtain the expected crystal habit. These methods can produce high-quality crystals but do not consider the characteristics of seeds. Seeding is a high-efficient method for stabilizing batch crystallization and decreasing batch-to-batch variations. In this regard, operation conditions, especially seeding condition and supersaturation, were investigated in the present work. In seeded crystallization, supersaturation could be kept at a low level due to the consumption of seed growth. As a result, the process could be stabilized. In 2001, Lewiner et al. [7] published a paper regarding the influence of seeding parameters such as cooling rate of seeded slurries, temperature of introduction of seeds, and amount of seeds. Most researchers have studied the control of crystal habit and supersaturation for cooling or anti-solvent crystallization, but few papers have been reported for reactive crystallization. In the pharmaceutical industry, reactive crystallization is an important operation for producing active pharmaceutical ingredients [8]. However, crystal shape control in reactive crystallization has been rarely investigated. Reactive 
crystallization differs from other crystallization methods due to its unique features and operation processes. Moreover, studies on reactive crystallization were mainly carried out on inorganic materials rather than organic molecules.

Cephalexin, a first-generation cephalosporin, has low toxicity and good oral absorption and is widely used in clinical practice [8]. The cephalexin crystal has poor crystal habit and shape, which lead to poor density and downstream processability. However, few articles have been reported on the crystallization process of cephalexin. In this regard, scholars have focused on analysis of the reactive crystallization system, particularly on the relationships between crystallization process and improvement of product quality (such as crystal habit, aspect ratio and final crystal size distribution).

The aim of this work is to control the crystal habit and shape by comparing a series of operation conditions through batch reactive crystallization. First, the solubility of cephalexin in water at different temperatures and $\mathrm{pH}$ levels was determined through laser monitoring. Second, different products were obtained by controlling supersaturation and optimizing the seeding condition by batch reactive crystallization. The products obtained under different operation conditions were compared through particle characterization methods using imaging instrument Morphologi G3 and Mastersizer 3000. The aspect ratio of the products was optimized. The crystal habit was changed from needle-like to plate-like through seed-assisted reactive crystallization, and the crystal shape was improved from an aspect ratio of 0.189 (Run S04) to 0.624 (Run S09). Supersaturation control and seeding conditions for the operations were given emphasis. Finally, the quality of the products was optimized by combining particle characterization methods using imaging instrument Morphologi G3 and Mastersizer 3000.

\section{Materials and Methods}

\section{Materials}

Cephalexin was supplied by Hebei Pharmacy Technology Co., Ltd., Shijiazhuang, China. Analytical-grade sulfuric acid (mass purity of $>98 \%$ ) was obtained from Tianjin Kewei Chemical Reagent Co, Tianjin, China. Sodium hydroxide (mass purity of $>96 \%$ ) was acquired from Tianjin Jiangtian Chemical Reagent Co. All chemicals were used directly as supplied by the manufacturers without further purification. Pure water, with a resistivity of $18.2 \mathrm{M} \Omega \mathrm{cm}$, was produced by NANOPURE system from BARNSTEAD (Thermo Scientific Co., China) and used to prepare solutions. The main parameters are presented in Table 1.
Table 1 Main parameters of instruments used in this paper

\begin{tabular}{ll}
\hline Measurement control & Analysis of 1 glass plate \\
Sample carrier & Glass plate $180 \mathrm{~mm} \times 110 \mathrm{~mm}$ \\
Sample mass & $\sim 20-40 \mathrm{mg}$ \\
SDU settings & Injection pressure $3 \mathrm{bar}$ \\
& Injection time $10 \mathrm{~ms}$ \\
& Settling time $60 \mathrm{~s}$ \\
Compensation for tilts & Enabled \\
Illumination & Diascopic \\
Optics selection & $5 \times$ \\
Overlap & $40 \%$ \\
Focus & Manual focus \\
Threshold & $0-105$ \\
Scan area & $5330 \mathrm{~mm}^{2}$ \\
Trash size & 50 pixels \\
Filters & CED $<80$ \\
\hline
\end{tabular}

\section{Methods}

Solubility of cephalexin at different $\mathrm{pH}$ levels and temperatures was determined using the method of laser monitoring. This method was used to monitor the dissolution of solutes at a fixed temperature. The system included a photoelectric transformer, a laser generator, and a light intensity display.

Water $(30 \mathrm{~g})$ and a certain amount of $1 \mathrm{~mol} / \mathrm{L}$ sulfuric acid solution (or $25 \%$ mass concentration ammonia solution) were placed in a jacketed glass vessel (about $50 \mathrm{~mL}$ ). The $\mathrm{pH}$ of the solution was adjusted to a desired value. The mass of all reagents was determined using an analytical balance (type AB204, Mettler-Toledo, Switzerland). The temperature of the system was controlled by thermostatic water bath (type MPG-10C, China), with a standard uncertainty of $0.1 \mathrm{~K}$. The intensity of the laser beam started at the lowest point owing to undissolved cephalexin. As the experiment proceeded, the intensity of the laser beam increased to the peak level because of dissolution until the solution became transparent. Cephalexin of known mass (around $0.0020 \mathrm{~g}$ ) was then added and recorded. After the final addition of cephalexin, the maximum intensity of the laser beam could not reach $90 \%$ of the initial value. The final $\mathrm{pH}$ and total mass of the solute were recorded. The interval time was usually no less than $40 \mathrm{~min}$. The total mass of the solute added was recorded as $m_{1}$, the total amount of water (water in sulfuric acid solution included) was recorded as $m_{2}$, and the total amount of sulfuric acid was recorded as $m_{3}$. The experiment was repeated three times, and the arithmetic average of $x_{1}$ was taken as the final experimental value to ensure accuracy.

The mole fraction solubility of cephalexin $\left(x_{1}\right)$ in monosolvents is calculated by Eq. (1). 
$x_{1}=\frac{m_{1} / M_{1}}{m_{1} / M_{1}+m_{2} / M_{2}+m_{3} / M_{3}}$

where $m_{1}, m_{2}$ and $m_{3}$ represent the masses of cephalexin, water, and sulfuric acid (or ammonia); $M_{1}, M_{2}$, and $M_{3}$ are the mole masses of cephalexin, water, and sulfuric acid (or ammonia), respectively.

Reactive unseeded crystallization was conducted in a 250-mL glass jacketed crystallizer. A propeller-type agitator was used to mix the reactive system with an agitation speed of $600 \mathrm{r} / \mathrm{min}$. For unseeded and seeded operations, the initial concentration was around $13 \%$ (kilogram of cephalexin/ kilogram of slurry). The solution was kept in a $250-\mathrm{mL}$ feed tank, and the crystallizer and feed tank were maintained at a certain temperature by the circulating water through the outer jacket from the thermostatic water bath. Before each reaction, $40 \mathrm{~mL}$ of water was injected into the crystallizer for initial mixing.

Seeded batch operations are similar to unseeded batch operations except for the addition of seeds at a certain time and the supersaturation before the appearance of primary nucleation.

Experiments were carried out at different feeding rates (340-613 $\mu \mathrm{L} / \mathrm{min}$ ) in the unseeded operation. In seedassisted crystallization, various seeding supersaturation levels $(S=1.0-1.8)$, amount of seeds $(0-10 \%)$, and feeding rates $(340-511 \mu \mathrm{L} / \mathrm{min})$ were used. The process and equipment are shown in Fig. 1.

\section{Image-Based Particle Characterization}

Particle size analysis was performed with a Morphologi G3 particle characterization system (Malvern Instruments, Malvern, UK). The system can be used for automated analysis

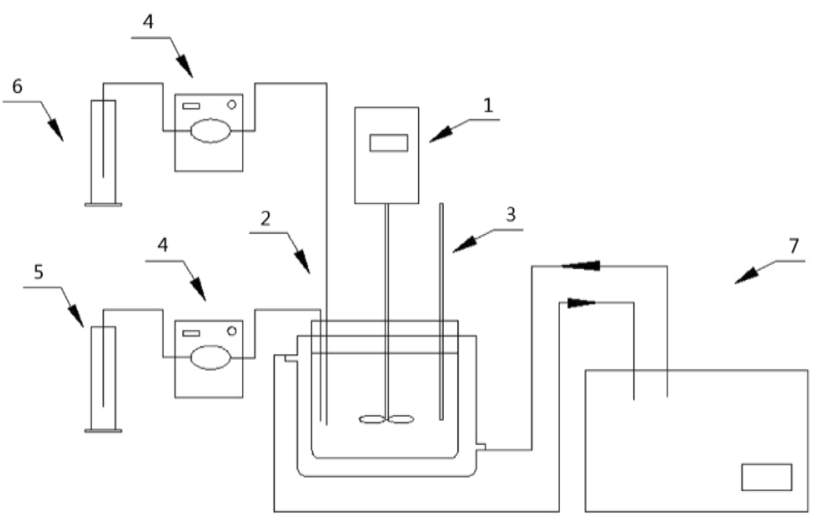

Fig. 1 A schematic representation of the crystallizer used for the present study. $1-$ propeller-type agitator; 2 -crystallizer; $3-\mathrm{pH}$ meter; 4-peristaltic pump; 5-base; 6-crystallization solution; 7-thermostatic bath of two-dimensional images of three-dimensional particles. Typical parameters (length $L$, width $W$, and aspect ratio) were chosen as references in this research. These size and shape parameters were calculated for each grain, from which mean values and standard deviations were extracted to characterize each sample. Aspect ratio is the ratio of the width to the length of the particle and is calculated as:

Aspect Ratio $=\frac{\text { Width }}{\text { Length }}$

The aspect ratio values are within 0 to 1 .

\section{UV-Vis Spectrophotometer}

An ultraviolet spectrophotometer was employed for offline determination of solution concentration. For traditional UV-Vis measurement, the aqueous solution of cephalexin was placed in a quartz cuvette and analyzed on a U3010 $\mathrm{UV}-\mathrm{V}$ is spectrophotometer. UV-Vis absorbance spectra were scanned within the wavelength range of 191-1100 nm with $2 \mathrm{~nm}$ increments. The $253 \mathrm{~nm}$ wavelength was selected for recording of the absorbance of the diluted solutions. The calibration curves for determining the concentrations of each liquid in diluted solutions of water were prepared. The crystallization solutions were filtered by a water membrane $(0.22 \mu \mathrm{m}$, Tianjin Legg Technology Co., Ltd, Tianjin, China). The filtrate was diluted with water and analyzed by UV-Vis. The concentrations of the solution were calculated based on the Lambert-Beer law calibration curve.

\section{Measurement of Bulk Density}

Bulk density was determined by pouring the products of cephalexin into a 50-mL graduated cylinder by free falling without any further vibration or compaction. The total amount of the solute added into the graduated cylinder was recorded as $m$, and the volume was recorded as $V$. The experiment was repeated three times, and the arithmetic average of $\rho$ was taken as the final experimental value to ensure accuracy.

The bulk density of cephalexin $(\rho)$ is calculated by Eq. (2).

$\rho=\frac{m}{V}$

Density without further explanation in the following was short for bulk density. 


\section{Results and Discussion}

\section{Solubility of Cephalexin}

The experimental solubility data of cephalexin in water and aqueous electrolyte solutions at different $\mathrm{pH}$ levels and temperatures are shown in Table 2 and Fig. 2.

Tsuji et al. [9] determined the solubility of cephalexin monohydrate of different $\mathrm{pH}$ levels at $37{ }^{\circ} \mathrm{C}$, and the result is similar to the experimental value obtained at $308.15 \mathrm{~K}$ $\left(35^{\circ} \mathrm{C}\right)$ in the present study; hence, the solubility data are of high accuracy.

The solubility of cephalexin in pure water is very low and increases slightly with increasing temperature; as such, cooling crystallization is neither efficient nor economical. Meanwhile, the solubility changes dramatically when the $\mathrm{pH}$ is lower than 3.0 or higher than 7.0 due to the dissociation of cephalexin. Thus, reactive crystallization or isoelectric point crystallization has high efficiency. Cephalexin is not as stable in the alkaline environment as in the acidic environment. In the present work, reactive crystallization in acidic conditions was chosen because this method exhibits high efficiency and economical value and is also widely used for crystallization of cephalexin in industries.

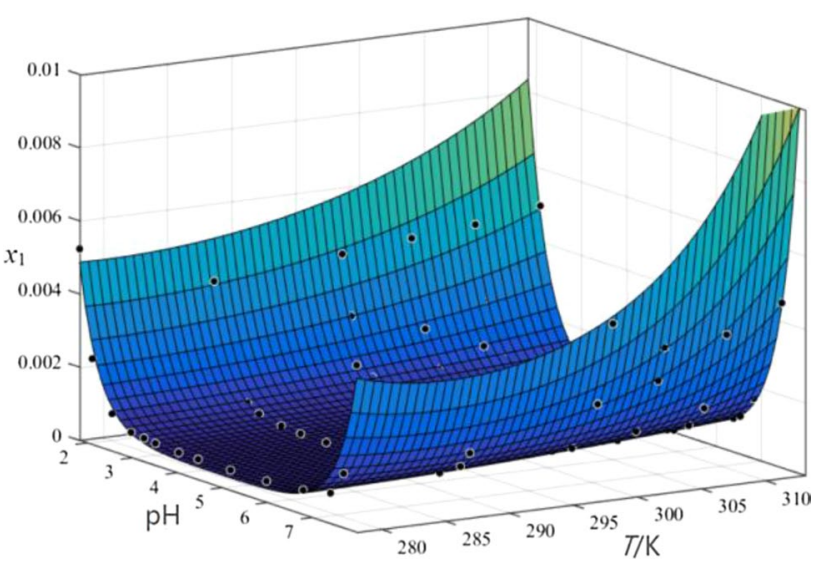

Fig. 2 Mole fraction solubility $\left(x_{1}\right)$ of cephalexin versus the $\mathrm{pH}$ and temperature in aqueous solution [experimental solubility; surface is calculated by Eq. (1)]

\section{Conditions of Preliminary Experiments}

To investigate the effect of $\mathrm{pH}$ and temperature on supersaturation and aspect ratio, many preliminary experiments were conducted.

In unseeded experiments, the metastable zone near the isoelectric point was very narrow and prone to spontaneous nucleation and formation of needle-like crystals. In seeding experiments, the same phenomenon was observed. Linear experiments were carried out under different $\mathrm{pH}$ levels between 3.26 and 4.4 (around the isoelectric point, the specific parameters are shown in Table $\mathrm{S} 1$ ). When the $\mathrm{pH}$ is
Table 2 Mole fraction solubility of cephalexin in aqueous solution at different temperatures $(0.1 \mathrm{MPa})$

\begin{tabular}{|c|c|c|c|c|c|c|c|c|c|}
\hline \multicolumn{2}{|c|}{$278.15 \mathrm{~K}$} & \multicolumn{2}{|c|}{$288.15 \mathrm{~K}$} & \multicolumn{2}{|c|}{$298.15 \mathrm{~K}$} & \multicolumn{2}{|c|}{$303.15 \mathrm{~K}$} & \multicolumn{2}{|c|}{$308.15 \mathrm{~K}$} \\
\hline $\mathrm{pH}$ & $10^{3} x_{1}$ & $\mathrm{pH}$ & $10^{3} x_{1}$ & $\mathrm{pH}$ & $10^{3} x_{1}$ & $\mathrm{pH}$ & $10^{3} x_{1}$ & $\mathrm{pH}$ & $10^{3} x$ \\
\hline 7.98 & 4.56 & 7.96 & 4.63 & 7.98 & 4.81 & 7.70 & 3.82 & 7.66 & 3.93 \\
\hline 7.69 & 1.48 & 7.65 & 1.58 & 7.64 & 2.47 & 7.56 & 2.86 & 7.16 & 1.72 \\
\hline 7.40 & 0.826 & 7.44 & 1.13 & 7.07 & 1.03 & 7.07 & 1.27 & 6.82 & 1.11 \\
\hline 6.79 & 0.662 & 6.98 & 0.763 & 6.63 & 0.776 & 6.66 & 0.856 & 6.49 & 0.846 \\
\hline 5.98 & 0.578 & 6.59 & 0.662 & 6.05 & 0.639 & 6.32 & 0.692 & 6.34 & 0.796 \\
\hline 5.18 & 0.552 & 6.27 & 0.622 & 5.50 & 0.610 & 6.02 & 0.658 & 5.98 & 0.678 \\
\hline 4.46 & 0.557 & 5.85 & 0.582 & 4.94 & 0.572 & 5.67 & 0.631 & 4.57 & 0.626 \\
\hline 4.03 & 0.561 & 5.08 & 0.556 & 4.40 & 0.576 & 4.89 & 0.604 & 4.37 & 0.638 \\
\hline 3.51 & 0.604 & 4.46 & 0.570 & 4.11 & 0.577 & 4.29 & 0.628 & 4.02 & 0.636 \\
\hline 3.26 & 0.644 & 3.89 & 0.578 & 3.69 & 0.635 & 3.84 & 0.643 & 3.42 & 0.737 \\
\hline 2.97 & 0.684 & 3.46 & 0.622 & 3.26 & 0.737 & 3.34 & 0.736 & 2.92 & 0.996 \\
\hline 2.55 & 1.02 & 2.97 & 0.749 & 2.92 & 0.888 & 2.98 & 0.908 & 2.59 & 1.47 \\
\hline 2.54 & 1.03 & 2.71 & 0.929 & 2.64 & 1.16 & 2.69 & 1.18 & 2.69 & 1.29 \\
\hline 2.11 & 2.35 & 2.08 & 2.39 & 2.17 & 2.65 & 2.39 & 2.17 & 2.26 & 2.66 \\
\hline 1.83 & 5.23 & 1.97 & 3.96 & 1.97 & 4.26 & 2.09 & 4.52 & 2.08 & 4.67 \\
\hline
\end{tabular}

The relative standard uncertainty of the solubility measurement is $\operatorname{Ur}\left(x_{1}\right)=0.05$; the relative uncertainty of temperature is $\mathrm{Ur}(T)=0.05 \mathrm{~K}$; the relative uncertainty of $\mathrm{pH}$ is $\mathrm{Ur}(\mathrm{pH})=0.01$; the relative uncertainty of pressure is $\operatorname{Ur}(p)=0.01$ 
increased from 3.26 to 4.4 , the theoretical yield increases by $2.5 \%$ from 88.5 to $91 \%$, whereas the bulk density decreases by $27 \%$ from 0.26 to $0.19 \mathrm{~g} / \mathrm{cm}^{3}$. The crystal shape tends to be needle-like (Fig. S1). Therefore, we fixed the $\mathrm{pH}$ at 3.26 as the crystallization point to control supersaturation. In addition, the change in solubility with temperature is not obvious from the solubility curve of cephalexin, and the metastable zone width has no obvious change with temperature. Thus, $298 \mathrm{~K}\left(25^{\circ} \mathrm{C}\right)$ was chosen as the crystallization temperature.

\section{Unseeded Batch Operations}

For process control, complex operating parameters, such as feeding rate or seeding condition, should be handled in industrial chemistry. As such, unseeded batch operations were carried out first to investigate the effect of feeding rate of the reactant on the supersaturation $S$ and on the aspect ratio of the final product. (The operating parameters are presented in Table 3.)

Different feeding rates lead to different crystal aspect ratios (Figs. 3, 4). A lower feeding rate leads to a higher aspect ratio.

To some extent, nucleation concentration was difficult to be controlled efficiently by controlling feeding rate (Table 3; runs N02, N03, and N04). Random primary nucleation occurred in unseeded operations, resulting in batch-to-batch variations.

The aspect ratio of all products is low during unseeded operation, and the crystal habit is fine needle-like, which is undesirable in industrial applications (Fig. 5).

Moreover, the concentration of the solvents during unseeded operation decreased rapidly after reaching "a peak," leading to quick nucleation. Therefore, we can improve the operation reproducibility by seeding before nucleation. Subsequently, seed-assisted batch operations were carried out.

Table 3 Operating parameters of unseeded reactive crystallizations of cephalexin

\begin{tabular}{lllrll}
\hline No. & $\begin{array}{l}\text { Feeding rate } \\
(\mu \mathrm{L} / \mathrm{min})\end{array}$ & $L(\mu \mathrm{m})$ & $W(\mu \mathrm{m})$ & $W / L$ & $\rho\left(\mathrm{g} / \mathrm{cm}^{3}\right)$ \\
\hline N01 & 613 & 36.6 & 5.82 & 0.159 & 0.1 \\
N02 & 438 & 46.19 & 7.62 & 0.165 & 0.111 \\
N03 & 368 & 43.14 & 8.24 & 0.191 & 0.13 \\
N04 & 340 & 49.95 & 12.22 & 0.253 & 0.168 \\
\hline
\end{tabular}

The initial concentration was around $13 \%$ ( $\mathrm{kg}$ cephalexin/kg slurry); the crystallization $\mathrm{pH}$ value was 3.26 ; and the crystallization temperature was $298 \mathrm{~K}$

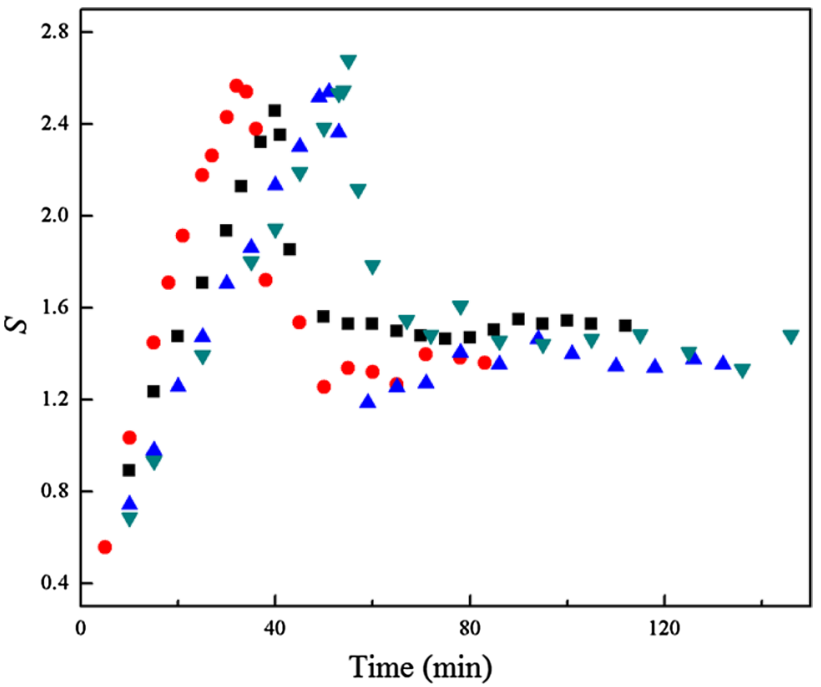

Fig. 3 Supersaturation profiles of dissolved cephalexin measured during unseeded batch reactive crystallizations in water (feeding rate: $613 \mu \mathrm{L} / \mathrm{min} ; 438 \mu \mathrm{L} / \mathrm{min} ; 368 \mu \mathrm{L} / \mathrm{min} ; 340 \mu \mathrm{L} / \mathrm{min}$ )

\section{Seed-Assisted Batch Operation}

The products turned into rod-like or mixtures of needle-like and rod-like crystals when seeds were used relative to the needle-like crystals obtained in unseeded crystallization. Seeding supersaturation, mass of seeds, and feeding rate were investigated in this section to determine the influence of different seeding conditions on aspect ratio.

A series of experiments was carried out and reported in the following.

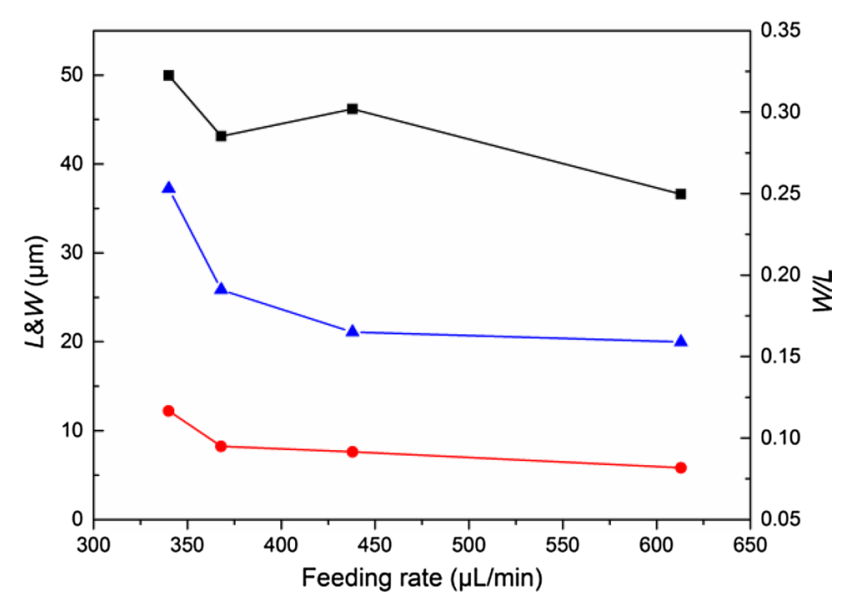

Fig. 4 Dependency of length, width and aspect ratio on feeding rate in unseeded operations ( $\square$ length; width; aspect ratio) 
Fig. 5 Microscope images of cephalexin obtained from different experiments conditions. a Particles for unseeded batch operations; $\mathbf{b}$ particles for optimal experiment conditions in seeded batch operations
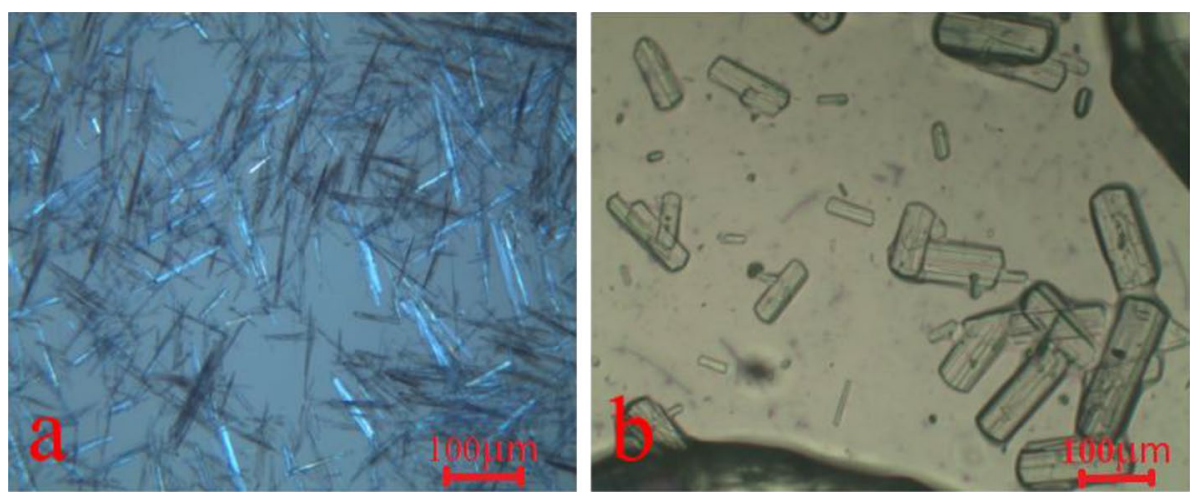

Table 4 Operating parameters of seeded reactive crystallizations of cephalexin

\begin{tabular}{llllllll}
\hline No. & $\begin{array}{l}\text { Seeding super- } \\
\text { saturation }\end{array}$ & $\begin{array}{l}\text { Seed mass } \\
\text { ratio }(\%)\end{array}$ & $\begin{array}{l}\text { Feeding rate } \\
(\mu \mathrm{L} / \mathrm{min})\end{array}$ & $L(\mu \mathrm{m})$ & $W(\mu \mathrm{m})$ & $W / L$ & $\begin{array}{l}\text { Coefficient } \\
\text { of variation }\end{array}$ \\
\hline S01 & 1.0 & 8 & 438 & 57.63 & 17.35 & 0.301 & 0.184 \\
S02 & 1.3 & 8 & 438 & 76.24 & 26.8 & 0.352 & 0.192 \\
S03 & 1.5 & 8 & 438 & 74.93 & 25.7 & 0.343 & 0.194 \\
S04 & 1.8 & 8 & 438 & 43.44 & 8.21 & 0.189 & 0.128 \\
S05 & 1.3 & 2 & 368 & 56.58 & 14.88 & 0.263 & 0.173 \\
S06 & 1.3 & 4 & 368 & 73.6 & 23.11 & 0.314 & 0.21 \\
S07 & 1.3 & 6 & 368 & 68.62 & 30.26 & 0.441 & 0.28 \\
S08 & 1.3 & 8 & 368 & 64 & 34.75 & 0.543 & 0.319 \\
S09 & 1.3 & 10 & 368 & 59.66 & 37.23 & 0.624 & 0.339 \\
S10 & 1.3 & 8 & 511 & 78.53 & 26.9 & 0.349 & 0.223 \\
S11 & 1.3 & 8 & 400 & 67.87 & 29.32 & 0.422 & 0.274 \\
S12 & 1.3 & 8 & 340 & 72.66 & 33.28 & 0.458 & 0.289 \\
\hline
\end{tabular}

The initial concentration was around $13 \%$ (kg cephalexin/kg slurry); the crystallization $\mathrm{pH}$ value was 3.26; and the crystallization temperature was $298 \mathrm{~K}$

\section{Effect of Seeding Supersaturation}

The effect of seeding supersaturation $S$ (from 1.0 to 1.8) was investigated (Table 4, runs S01 to S04). The mass of seeds was kept at $8 \%$ of the mass of final products, and the feeding rate was also kept constant at $438 \mu \mathrm{L} / \mathrm{min}$.

The concentration of cephalexin was monitored using an ultraviolet spectrophotometer. Supersaturation profiles were calculated and are shown in Fig. 6. The solution reached saturation at a feeding time of $10 \mathrm{~min}$, and different seeding times led to different changes in concentration. Compared with the unseeded operation, the addition of seeds decreased the maximum supersaturation from $S=2.5$ to lower than $S=2.2$. A less sharp decrease occurred after the concentration reached the peak, indicating that the burst of nucleation was avoided to some extent. In runs S01, S02, S03, and S04, the seeds were added at supersaturation $S=1.0, S=1.3$, $S=1.5$, or $S=1.8$, respectively. When seeds were added at around $S=1.5$ or earlier, no significant peak of supersaturation appeared, indicating that crystallization was carried out in a relatively stable condition. Under this condition, contact

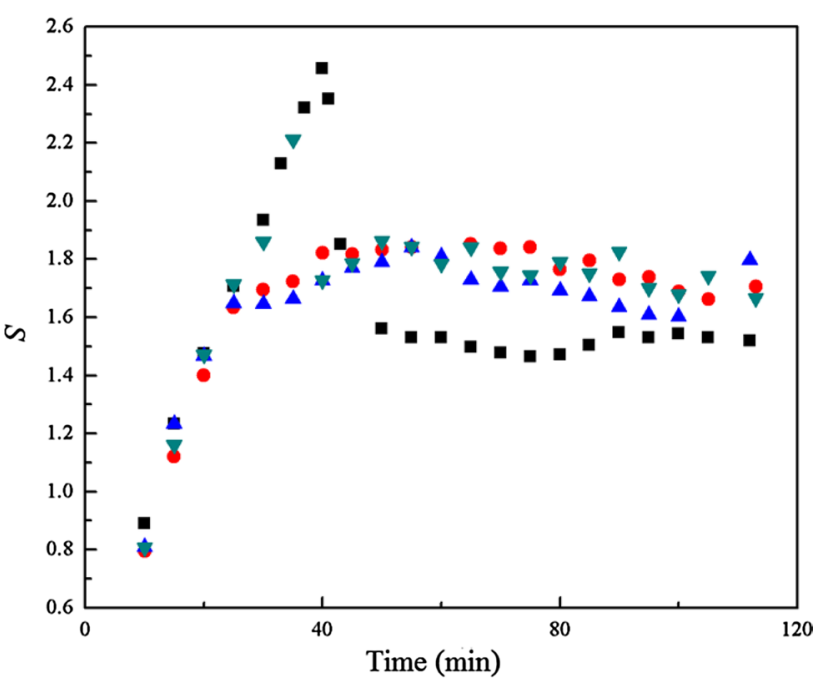

Fig. 6 Supersaturation profiles of different seeding supersaturation levels measured during seeded batch reactive crystallizations unseeded; $S=1.0 ; \quad S=1.3 ; \quad S=1.8$ ) 
nucleation and growth dominated, and the products are uniform and have good shape (plate-like crystals). When the seeds were added at around $S=1.8$ or later, sharp decrease in the supersaturation appeared after the peak, indicating the occurrence of a burst of primary nucleation and contact nucleation; the formed products are needle-like and platelike mixtures. This result was supported by the analysis of aspect ratio (Fig. 7); that is, improved aspect ratio and platelike crystals were obtained at intermediate values of seeding time when the supersaturation is around 1.3. The following experiments were carried out at $S=1.3$.

\section{Effect of Mass of Seeds}

The effect of the mass of seeds on the aspect ratio was determined by investigating the supersaturation profiles and density of the final products. The feeding rate and seeding supersaturation level were kept constant at $368 \mu \mathrm{L} / \mathrm{min}$ and $S=1.3$ (seeding time is $18 \mathrm{~min}$ ), respectively. The mass of the seeds was set between 2 and $10 \%$ of the mass of the final products.

As the mass ratio of the seeds increased, the maximum supersaturation or the peak of supersaturation decreased (Fig. 8). When the mass ratio of the seeds reached $8 \%$, no peak was observed. At the same time, the aspect ratio increased and the crystal habit turned into plate-like, indicating that sufficient crystal surfaces were provided so that contact nucleation and growth dominated under a relative stable condition. When the mass ratio was $2 \%$, a sudden decrease in the supersaturation level occurred, resulting in burst nucleation and formation of fine needle-like products. This result is shown in Fig. 9; that is, the more seeds used, the better the aspect ratio will be.

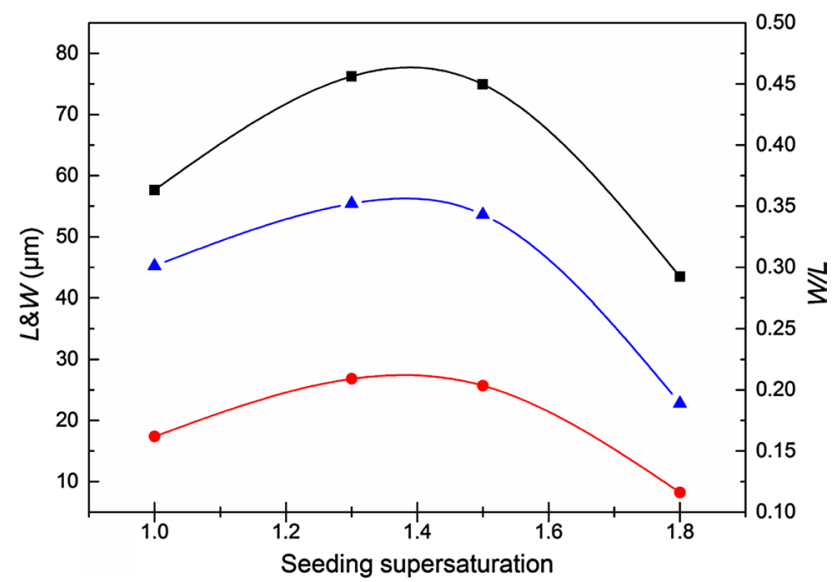

Fig. 7 Dependency of length, width and aspect ratio on seeding supersaturation in seeded operations $(\square$ length; $\boldsymbol{w}$ width; aspect ratio)

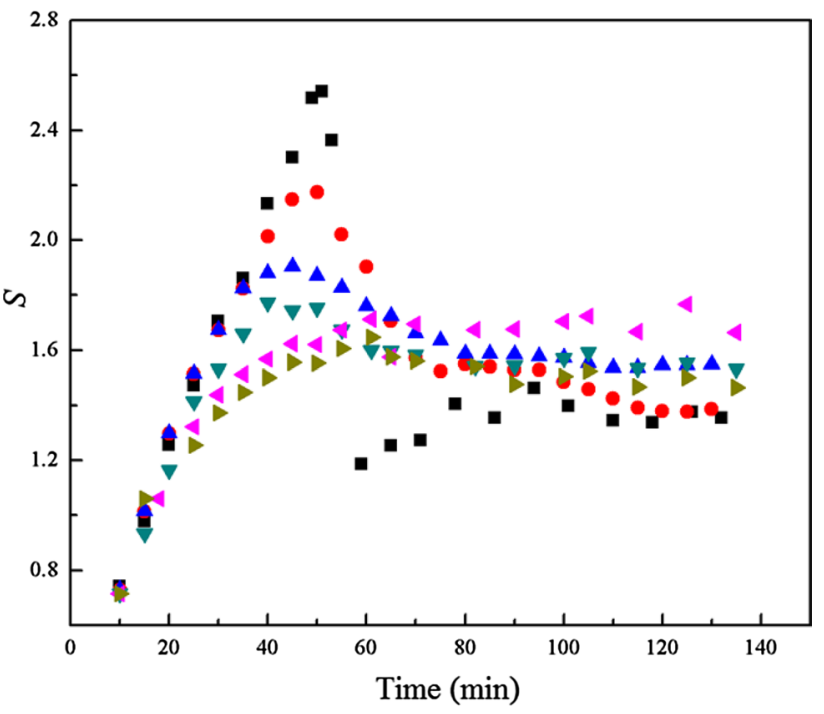

Fig. 8 Supersaturation profiles in seeded crystallization under different seed mass ratios $(\square$ unseeded; $2 \% ; 4 \% ; \quad 6 \% ; 8 \%$; $10 \%)$

A large amount of seeds should be applied to batch operation to obtain products with better shape. However, the cost of seeds must be considered in industrial production; in this regard, we selected $8 \%$ as the final mass of seeds for subsequent experiments.

The influence of the mass of seeds on particle size was investigated in two simulation papers. Bohlin and Rasmuson [10] concluded that as the mass of seeds increases, the mean size of the final products may increase or decrease; this finding is similar to the results of the present work. In a word, the effect of the mass of seeds may be explained due to the domination of secondary nucleation mechanisms [11].

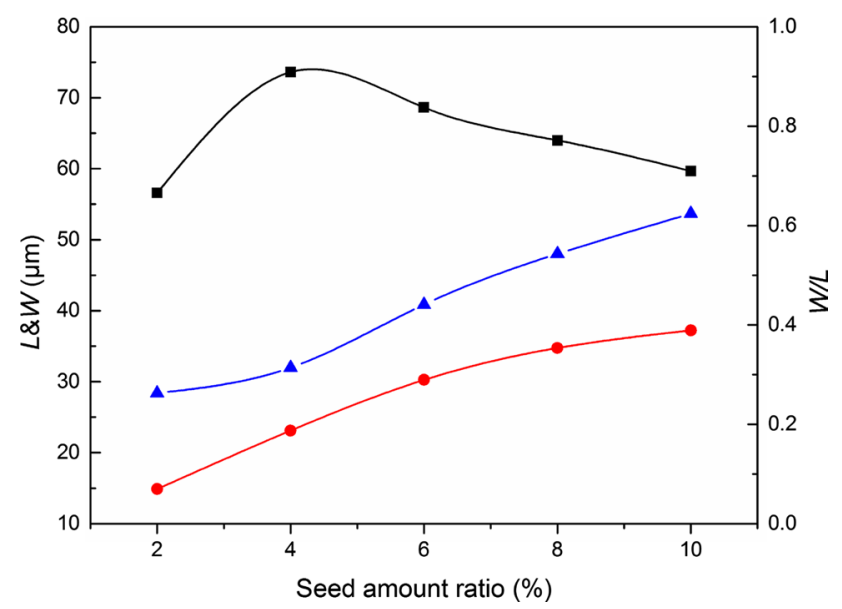

Fig. 9 Dependency of length, width and aspect ratio on seed amount ratio ( length; width; aspect ratio) 


\section{Effect of Feeding Rate}

Several batch operation experiments (Table 4; runs S08, S10, S11, and S12) were conducted with different feeding rates (340-511 $\mu \mathrm{L} / \mathrm{min}$, see Figs. 10 and 11). The mass of seeds was kept to $8 \%$ of the mass of the final products, and the seeding supersaturation level was maintained at $S=1.3$.

We observed the crystal nucleation and growth by process sampling. Run S10 (with a feeding rate of $511 \mu \mathrm{L} / \mathrm{min}$ ) was taken as an example; the images of the crystal in the process are shown in Fig. 12. At 30 min, supersaturation contributed mainly to crystal growth, accompanied by minimal secondary nucleation, so the crystals are plate-like. At $50 \mathrm{~min}$, the rate of supersaturation consumption decreased, resulting in the production of needle-like crystals. When the ammonia solution was added, supersaturation continuously accumulated and burst nucleation occurred after 70 min, leading to the generation of a large number of needle-like crystals.

The supersaturation profiles and the optical microscope images are shown in Figs. 5 and 10. The crystals obtained at a low feeding rate are plate-like and have high aspect ratio (Table 4) because of the gentle supersaturation curve and suppressed primary nucleation. When the feeding rate is too slow $(340 \mu \mathrm{L} / \mathrm{min})$, the aspect ratio and bulk density decreases because the long axis of crystals grows faster in lower supersaturation level.

The morphological variations observed in the experiments were caused by different supersaturation levels. The reason for the supersaturation effect on crystal habit has been studied. In different supersaturation levels, the growth mechanisms for a flat $(F)$ face differ [6]. Bordawekar et al. [6] investigated the effects of supersaturation on the crystal habit

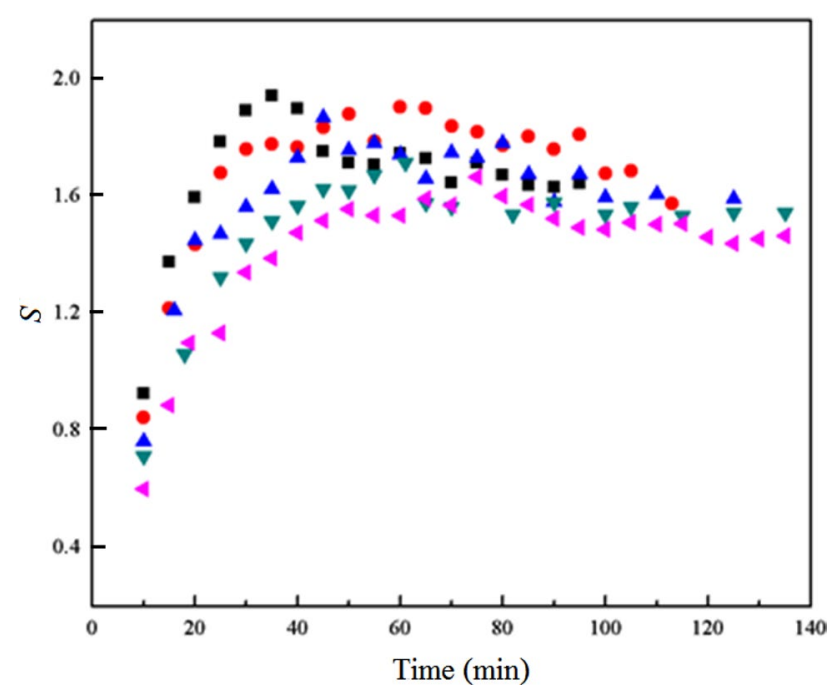

Fig. 10 Supersaturation profiles measured in seeded crystallization under different feeding rates $(511 \mu \mathrm{L} / \mathrm{min} ; 438 \mu \mathrm{L} / \mathrm{min}$; $400 \mu \mathrm{L} / \mathrm{min} ; 368 \mu \mathrm{L} / \mathrm{min} ; 340 \mu \mathrm{L} / \mathrm{min})$ of choline fenofibrate and provided mechanistic insights into the crystal habit; the spiral growth model was used for morphology prediction of organic molecular crystals to explain the effect of supersaturation on the crystal habit of choline fenofibrate based on the concepts of stability of surfaces, building units, periodic bond chain theory, and spiral growth model. In our investigation, the influence of supersaturation agreed well with the conclusions of Bordawekar et al.

The average size of the products measured by Morphologi G3 significantly increased with increasing feeding rate. As such, we assume that the seeding supersaturation investigated in this paper lies inside the metastable zone of the crystallization system, and an excessive increment in the feeding rate leads to the increment in supersaturation to the metastable zone; subsequently, the secondary "true" nucleation will occur, producing smaller products (Figs. 11, 12).

Based on the results of UV measurements on supersaturation combined with Malvern Morphologi G3 on the aspect ratio, the feeding rate of $368 \mu \mathrm{L} / \mathrm{min}$ was chosen as the optimal experimental condition.

\section{Conclusions and Perspectives}

In batch reactive crystallization of cephalexin in acidic aqueous solution, determinations of supersaturation by UV and aspect ratio by Morphologi G3 could be used to investigate the process of the system. In unseeded crystallization, the process is difficult to control due to the primary burst nucleation and the ungentle supersaturation curve after primary burst nucleation.

In this research, seeding is proved to be a highly efficient method for increasing the average aspect ratio of products. Notably, the possibility of increasing the average aspect ratio by optimal seeding policies is underlined. A satisfactory

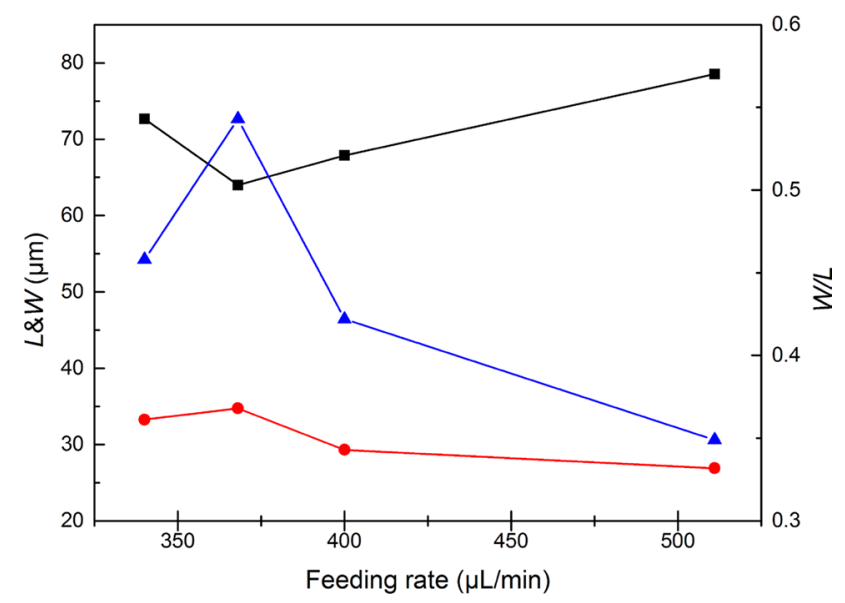

Fig. 11 Dependency of length, width and aspect ratio on feeding rate length; width; aspect ratio) 
Fig. 12 Images of crystal variation in the process for feeding rate of $511 \mu \mathrm{L} / \mathrm{min}(90-\mathrm{min}$ feeding time in total)
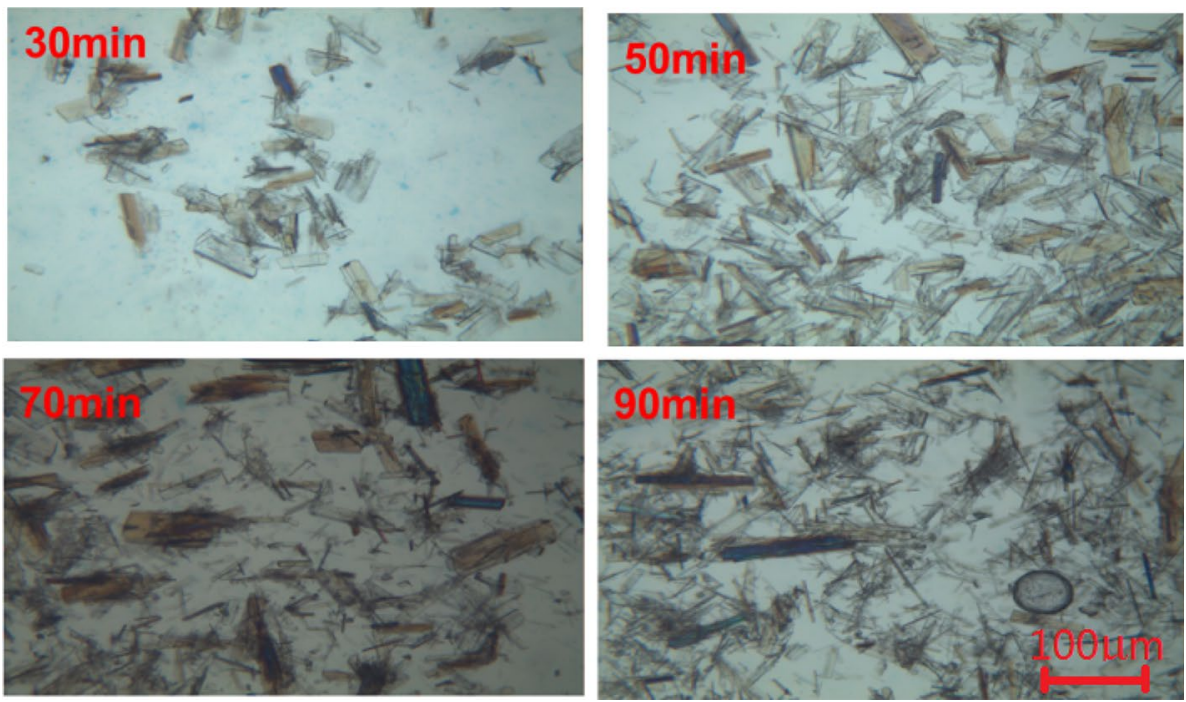

seeding policy including seeding supersaturation and seeding amount was investigated to improve the average aspect ratio. Different feeding rates were then investigated under the best seeding policy. A better feeding rate was obtained to improve the quality of products. The results could provide guidance for a better operation in industrial production. Nevertheless, theoretical works should be conducted due to the lack of research about seeding crystallization in organic compounds [12-14].

Acknowledgements This study was supported by the Major National Science and Technology Projects (No. 2017ZX07402003) and Major National Scientific Instrument Development Project (No. 21527812).

Open Access This article is distributed under the terms of the Creative Commons Attribution 4.0 International License (http://creativeco mmons.org/licenses/by/4.0/), which permits unrestricted use, distribution, and reproduction in any medium, provided you give appropriate credit to the original author(s) and the source, provide a link to the Creative Commons license, and indicate if changes were made.

\section{References}

1. Mullin JW (1992) Crystallization, 3rd edn. Butterworth-Heinemann, Oxford

2. Tavare NS (1995) Industrial crystallization. Plenum Press, New York

3. Kubota N, Doki N, Yokota M et al (2001) Seeding policy in batch cooling crystallization. Powder Technol 121(1):31-38
4. Borchert $\mathrm{C}$, Temmel E, Eisenschmidt $\mathrm{H}$ et al (2014) Image-based in situ identification of face specific crystal growth rates from crystal populations. Cryst Growth Des 14(3):952-971

5. Yang G, Kubota N, Sha Z et al (2006) Crystal shape control by manipulating supersaturation in batch cooling crystallization. Cryst Growth Des 6(12):2799-2803

6. Bordawekar S, Kuvadia Z, Dandekar P et al (2014) Interesting morphological behavior of organic salt choline fenofibrate: effect of supersaturation and polymeric impurity. Cryst Growth Des 14(8):3800-3812

7. Lewiner F, Fevotte G, Klein JP et al (2001) Improving batch cooling seeded crystallization of an organic weed-killer using on-line ATR FTIR measurement of supersaturation. J Cryst Growth 226(2-3):348-362

8. Barrett M, Hao H, Maher A et al (2011) In situ monitoring of supersaturation and polymorphic form of piracetam during batch cooling crystallization. Organ Process Res Dev 15(3):681-687

9. Tsuji A, Nakashima E, Hamano S et al (1978) Physicochemical properties of amphoteric $\beta$-lactam antibiotics I: stability, solubility, and dissolution behavior of amino penicillins as a function of pH. J Pharm Sci 67(8):1059-1066

10. Bohlin M, Rasmuson AC (1996) Importance of macromixing in batch cooling crystallization. AIChE J 42(3):691-699

11. Garside J (1982) Nucleation: biological mineralization and demineralization. Springer, Berlin, pp 23-35

12. Donnet M, Bowen P, Jongen N et al (2005) Use of seeds to control precipitation of calcium carbonate and determination of seed nature. Langmuir 21(1):100-108

13. Chung SH, Ma DL, Braatz RD (1999) Optimal seeding in batch crystallization. Can J Chem Eng 77(3):590-595

14. Bohlin M, Rasmuson AC (1992) Application of controlled cooling and seeding in batch crystallization. Can J Chem Eng 70(1):120-126 